\title{
Simple Finite Jordan Pseudoalgebras ${ }^{\star}$
}

Pavel KOLESNIKOV

Sobolev Institute of Mathematics, 4 Acad. Koptyug Ave., 630090 Novosibirsk, Russia

E-mail: pavelsk@math.nsc.ru

Received September 12, 2008, in final form January 10, 2009; Published online January 30, 2009 doi:10.3842/SIGMA.2009.014

\begin{abstract}
We consider the structure of Jordan $H$-pseudoalgebras which are linearly finitely generated over a Hopf algebra $H$. There are two cases under consideration: $H=U(\mathfrak{h})$ and $H=U(\mathfrak{h}) \# \mathbb{C}[\Gamma]$, where $\mathfrak{h}$ is a finite-dimensional Lie algebra over $\mathbb{C}, \Gamma$ is an arbitrary group acting on $U(\mathfrak{h})$ by automorphisms. We construct an analogue of the Tits-Kantor-Koecher construction for finite Jordan pseudoalgebras and describe all simple ones.
\end{abstract}

Key words: Jordan pseudoalgebra; conformal algebra; TKK-construction

2000 Mathematics Subject Classification: 17C50; 17B60; 16W30

\section{Introduction}

The notion of pseudoalgebra appeared as a natural generalization of the notion of conformal algebra. The last one provides a formal language describing algebraic structures underlying the singular part of the operator product expansion (OPE) in conformal field theory. Roughly speaking, the OPE of two local chiral fields is a formal distribution in two variables presented as $\sum_{n=-\infty}^{N-1} c_{n}(z)(w-z)^{-n-1}[3]$. The coefficients $c_{n}, n \in \mathbb{Z}$, of this distribution are considered as new "products" on the space of fields. The algebraic system obtained is called a vertex algebra. Its formal axiomatic description was stated in [4] (see also [7]). The "singular part" of a vertex algebra, i.e., the structure defined by only those operations with non-negative $n$, is a (Lie) conformal algebra [7].

Another approach to the theory of vertex algebras gives rise to the notion of a pseudotensor category [2] (which is similar to the multicategory of [14]). Given a Hopf algebra $H$, one may define the pseudotensor category $\mathcal{M}^{*}(H)$ [1] (objects of this category are left $H$-modules). An algebra in this category is called an $H$-pseudoalgebra. A pseudoalgebra is said to be finite if it is a finitely generated $H$-module.

In particular, for the one-dimensional Hopf algebra $H=\mathbb{k}$, $\mathbb{k}$ is a field, an $H$-pseudoalgebra is just an ordinary algebra over the field $\mathbb{k}$. For $H=\mathbb{k}[D]$, an $H$-pseudoalgebra is exactly the same as conformal algebra. In a more general case $H=\mathbb{k}\left[D_{1}, \ldots, D_{n}\right], n \geq 2$, the notion of an $H$-pseudoalgebra is closely related with Hamiltonian formalism in the theory of non-linear evolution equations [1]. For an arbitrary Hopf algebra $H$, an $H$-pseudoalgebra defines a functor from the category of $H$-bimodule associative commutative algebras to the category of $H$-module algebras (also called $H$-differential algebras).

An arbitrary conformal algebra $C$ can be canonically embedded in a "universal" way into the space of formal power series $A\left[\left[z, z^{-1}\right]\right]$ over an appropriate ordinary algebra $A[7,15]$. This algebra $A=\operatorname{Coeff} C$ is called the coefficient algebra of $C$. A conformal algebra is said to be associative (Lie, Jordan, etc.) if so is its coefficient algebra. For pseudoalgebras, a construction called annihilation algebra [1] works instead of coefficient algebra. However, the notion of

\footnotetext{
${ }^{\star}$ This paper is a contribution to the Special Issue on Kac-Moody Algebras and Applications. The full collection is available at http://www.emis.de/journals/SIGMA/Kac-Moody_algebras.html
} 
a pseudotensor category provides a direct way to the definition of what is a variety of pseudoalgebras [13].

In the paper [5], the complete description of simple finite Lie conformal algebras over $\mathbb{k}=\mathbb{C}$ was obtained. Apart from current conformal algebras, the only example of a simple finite Lie conformal algebra is the Virasoro conformal algebra. In the associative case, there are no exceptional examples: A simple finite associative conformal algebra is isomorphic to the current algebra over $M_{n}(\mathbb{C}), n \geq 1$. It was shown in [19] that there are no exceptional examples of simple finite Jordan conformal algebras.

In [1], the structure theory of finite Lie pseudoalgebras was developed. The classification theorem [1, Theorem 13.2] states that there exist simple finite Lie pseudoalgebras which are not isomorphic to current pseudoalgebras over ordinary simple finite-dimensional Lie algebras. This is not the case for associative pseudoalgebras. In this paper, we show the same for finite simple Jordan pseudoalgebras (Theorem 3): There are no examples of such pseudoalgebras except for current algebras (if $H=U(\mathfrak{h})$ ) or transitive direct sums of current algebras (if $H=U(\mathfrak{h}) \# \mathbb{C}[\Gamma])$. The main tool of the proof is an analogue of the well known Tits-KantorKoecher (TKK) construction for Jordan algebras. This result generalizes the classification of finite Jordan conformal algebras [19] to "multi-dimensional" case.

It was shown in [9] that the structure theory of Jordan conformal superalgebras is richer. The classification of simple finite Jordan superalgebras based on the structure theory of finite conformal Lie superalgebras [6] includes one series and two exceptional algebras [9, Theorem 3.9].

In our proof, we will not use annihilation algebras directly, the TKK construction will be built on the level of pseudoalgebras.

The paper is organized as follows. Section 2 contains the basics of Hopf algebras and pseudoalgebras theory, and some notations that will be used later. In Section 3, we introduce an analogue of the Tits-Kantor-Koecher construction (TKK) for finite Jordan pseudoalgebras. To complete the classification of finite simple Jordan pseudoalgebras, we need some technical results proved in Section 4. The main case under consideration is $H=U(\mathfrak{h})$, where $\mathfrak{h}$ is a finite-dimensional Lie algebra over $\mathbb{C}$. Another case is the smash-product $U(\mathfrak{h}) \# \mathbb{C}[\Gamma]$, where $\Gamma$ is an arbitrary group. These cases describe all cocommutative Hopf algebras over $\mathbb{C}$ with finite-dimensional spaces of primitive elements (see, e.g., [17]).

\section{Preliminaries on Hopf algebras and pseudoalgebras}

\subsection{Hopf algebras}

In this section, we state some notations that will be used later.

An associative algebra $H$ with a unit (over a field $\mathbb{k}$ ) endowed with coassociative coproduct $\Delta: H \rightarrow H \otimes H$ and counit $\varepsilon: H \rightarrow \mathbb{k}$ is called a bialgebra. Recall that both $\Delta$ and $\varepsilon$ are homomorphisms of algebras and

$$
(\mathrm{id} \otimes \Delta) \Delta(h)=(\Delta \otimes \mathrm{id}) \Delta(h), \quad(\varepsilon \otimes \mathrm{id}) \Delta(h)=(\mathrm{id} \otimes \varepsilon) \Delta(h)=h .
$$

To simplify the notation, we will use the following one which is due to Sweedler [17]: $\Delta^{[1]}(h):=h$, $\Delta^{[2]}(h):=\Delta(h)=\sum_{(h)} h_{(1)} \otimes h_{(2)}, \Delta^{[n]}(h):=\left(\operatorname{id} \otimes \Delta^{[n-1]}\right)=\sum_{(h)} h_{(1)} \otimes \cdots \otimes h_{(n)}$. Further, we will omit the symbol $\sum_{(h)}$ by writing $\Delta(h)=h_{(1)} \otimes h_{(2)}, \Delta^{[n]}(h)=h_{(1)} \otimes \cdots \otimes h_{(n)}$, etc.

Given a bialgebra $H$, an antihomomorphism $S: H \rightarrow H$ is called an antipode, if it satisfies

$$
S\left(h_{(1)}\right) h_{(2)}=\varepsilon(h)=h_{(1)} S\left(h_{(2)}\right) .
$$

A bialgebra with an antipode is called a Hopf algebra. 
There exists a natural structure of (right) $H$-module on the $n$th tensor power of $H$ (denoted by $\left.H^{\otimes n}\right)$ :

$$
\left(f_{1} \otimes \cdots \otimes f_{n}\right) \cdot h=f_{1} h_{(1)} \otimes \cdots \otimes f_{n} h_{(n)}, \quad f_{i}, h \in H .
$$

In this paper, we substantially consider cocommutative Hopf algebras, i.e., such that $h_{(1)} \otimes$ $h_{(2)}=h_{(2)} \otimes h_{(1)}$ for all $h \in H$. The antipode $S$ of a cocommutative Hopf algebra is involutive, i.e., $S^{2}=$ id.

For example, the universal enveloping algebra $U(\mathfrak{g})$ of a Lie algebra $\mathfrak{g}$ over a field of zero characteristic is a cocommutative Hopf algebra. Another series of examples is provided by the group algebra $\mathbb{k}[\Gamma]$ of an arbitrary group $\Gamma$ and by the general construction of smash-product. Namely, suppose $H$ is a Hopf algebra, and let a group $\Gamma$ acts on $H$ by algebra automorphisms. Then one may define the following new product on $H \otimes \mathbb{k}[\Gamma]$ :

$$
\left(h_{1} \otimes g_{1}\right) \cdot\left(h_{2} \otimes g_{2}\right)=h_{1} h_{2}^{g_{1}} \otimes g_{1} g_{2} .
$$

The algebra obtained is denoted by $H \# \mathbb{k}[\Gamma]$. Together with usual coproduct and antipode defined as on $H \otimes \mathbb{k}[\Gamma], H \# \mathbb{k}[\Gamma]$ is a Hopf algebra (the smash product of $H$ and $\mathbb{k}[G]$ ). If $H$ is cocommutative, then so is $H \# \mathbb{k}[\Gamma]$. Moreover, if $\mathbb{k}$ is an algebraically closed field of zero characteristic, then every cocommutative Hopf algebra $H$ over $\mathbb{k}$ is isomorphic to the smash product $U(\mathfrak{g}) \# \mathbb{k}[\Gamma]$ for appropriate $\mathfrak{g}$ and $\Gamma[16]$.

Lemma 1 ([1]). Let $H$ be a cocommutative Hopf algebra, and let $\left\{h_{i} \mid i \in I\right\}$ be a linear basis of $H$. Then every element $F \in H^{\otimes n+1}, n \geq 1$, can be uniquely presented as

$$
F=\sum_{i_{1}, \ldots, i_{n}}\left(h_{i_{1}} \otimes \cdots \otimes h_{i_{n}} \otimes 1\right) \cdot g_{i_{1}, \ldots, i_{n}}, \quad g_{i_{1}, \ldots, i_{n}} \in H
$$

In other words, the set $\left\{h_{i_{1}} \otimes \cdots \otimes h_{i_{n}} \otimes 1 \mid i_{1}, \ldots, i_{n} \in I\right\}$ is an $H$-basis of the $H$-module $H^{\otimes n+1}(2.1)$.

To find the presentation (2.2), one may use formal Fourier transformation $\mathcal{F}$ and its inverse $\mathcal{F}^{-1}[1]$ :

$$
\begin{aligned}
& \mathcal{F}, \mathcal{F}^{-1}: H^{\otimes n+1} \rightarrow H^{\otimes n+1} \\
& \mathcal{F}: h_{1} \otimes \cdots \otimes h_{n} \otimes f \mapsto h_{1} f_{(1)} \otimes \cdots \otimes h_{n} f_{(n)} \otimes f_{(n+1)}, \\
& \mathcal{F}^{-1}: h_{1} \otimes \cdots \otimes h_{n} \otimes f \mapsto h_{1} S\left(f_{(1)}\right) \otimes \cdots \otimes h_{n} S\left(f_{(n)}\right) \otimes f_{(n+1)} .
\end{aligned}
$$

We will use a "left" analogue of the Fourier transformation

$$
\mathcal{F}^{\prime}: h \otimes f_{1} \otimes \cdots \otimes f_{n} \mapsto h_{(1)} \otimes h_{(2)} f_{1} \otimes \cdots \otimes h_{(n+1)} f_{n},
$$

which is also invertible.

\subsection{Dual algebras}

Suppose $H$ is a cocommutative Hopf algebra, and let $X=H^{*}$ be its dual algebra (i.e., the product on $X$ is dual to the coproduct on $H$ ). Let us fix a linear basis $\left\{h_{i} \mid i \in I\right\}$ of $H$ and denote by $\left\{x_{i} \mid i \in I\right\} \subset X$ the set of dual functionals: $\left\langle x_{i}, h_{j}\right\rangle=\delta_{i j}, i, j \in I$.

An arbitrary element $x \in X$ can be presented as an infinite series in $x_{i}$ :

$$
x=\sum_{i \in I}\left\langle x, h_{i}\right\rangle x_{i}
$$


The algebra $X$ is a left and right module over $H$ with respect to the actions given by

$$
\langle x h, g\rangle=\langle x, g S(h)\rangle, \quad\langle h x, g\rangle=\langle x, S(h) g\rangle, \quad x \in X, h, g \in H .
$$

The actions (2.3) turn $X$ into a differential $H$-bimodule, i.e., $(x y) h=\left(x h_{(1)}\right)\left(y h_{(2)}\right), h(x y)=$ $\left(h_{(1)} x\right)\left(h_{(2)} y\right)$.

The operation $\Delta_{X}: X \rightarrow \overline{X \otimes X}:=(H \otimes H)^{*}$ dual to the product on $H$ is somewhat similar to a coproduct. From the combinatorial point of view, $\overline{X \otimes X}$ can be considered as the linear space of all infinite series $\sum_{i, j \in I} \alpha_{i j} x_{i} \otimes x_{j}, \alpha_{i j} \in \mathbb{k}$.

In order to unify notations, we will use $x_{(1)} \otimes x_{(2)}$ for $\Delta_{X}(x), x \in X$. In particular, the analogues of Fourier transforms

$$
\mathcal{F}: x \otimes y \mapsto x y_{(1)} \otimes y_{(2)}, \quad \mathcal{F}^{-1}: x \otimes y \mapsto x S^{*}\left(y_{(1)}\right) \otimes y_{(2)}, \quad x, y \in X,
$$

act from $X \otimes X$ to $\overline{X \otimes X}$.

Definition 1. Let $V$ be a linear space. A linear map $\pi: X \otimes X \rightarrow V$ is said to be local, if

$$
\pi\left(x_{i} \otimes x_{j}\right)=0 \quad \text { for almost all of the pairs } \quad(i, j) \in I^{2} .
$$

A local map $\pi: X \otimes X \rightarrow V$ can be naturally continued to $\bar{\pi}: \overline{X \otimes X} \rightarrow V$. The map $\bar{\pi}$ is continuous with respect to the topology on $\overline{X \otimes X}$ defined by the following family of basic neighborhoods of zero:

$$
U^{\perp}=\{\xi \in \overline{X \otimes X} \mid\langle\xi, U\rangle=0\}, \quad U \subseteq H \otimes H, \quad \operatorname{dim} U<\infty
$$

(we assume $V$ is endowed with discrete topology). Conversely, given a continuous linear map $\bar{X} \otimes X \rightarrow V$, its restriction to $X \otimes X$ is local.

For example, let us fix $h_{1}, h_{2} \in H$ and consider the map $x \otimes y \mapsto\left\langle x, h_{1}\right\rangle\left\langle y, h_{2}\right\rangle$. It is clear that this map is local. Obviously, every local map $\pi: X \otimes X \rightarrow V$ is actually the "evaluation" map

$$
\pi(x \otimes y)=\mathrm{ev}_{a}(x, y):=\left(\langle x, \cdot\rangle \otimes\langle y, \cdot\rangle \otimes \operatorname{id}_{V}\right)(a)
$$

for an appropriate $a \in H \otimes H \otimes V$.

Lemma 2. Suppose $\pi: X \otimes X \rightarrow V$ is a local map, and denote $\pi \mathcal{F}=\bar{\pi} \circ \mathcal{F}, \pi \mathcal{F}^{-1}=\bar{\pi} \circ \mathcal{F}^{-1}$. Then both $\pi \mathcal{F}$ and $\pi \mathcal{F}^{-1}$ are local, $\pi \mathcal{F}=0$ implies $\pi=0$, and $\pi \mathcal{F}^{-1}=0$ implies $\pi=0$.

Proof. Formally speaking, we can not use $\mathcal{F}^{-1}$ as an inverse of $\mathcal{F}$ since both $\mathcal{F}$ and $\mathcal{F}^{-1}$ are not defined on the entire space $\overline{X \otimes X}$. But it is straightforward to check (see also [1]) that

$$
\Delta_{X}(x)=x_{(1)} \otimes x_{(2)}=\sum_{i \in I} x S\left(h_{i}\right) \otimes x_{i}=\sum_{i \in I} x_{i} \otimes S\left(h_{i}\right) x, \quad x \in X,
$$

So

$$
\begin{aligned}
\pi \mathcal{F}\left(x_{i} \otimes x_{j}\right) & =\bar{\pi}\left(\sum_{k \in I} x_{i}\left(x_{j} S\left(h_{k}\right)\right) \otimes x_{k}\right)=\bar{\pi}\left(\sum_{k, l \in I}\left\langle x_{i}\left(x_{j} S\left(h_{k}\right)\right), h_{l}\right\rangle x_{l} \otimes x_{k}\right) \\
& =\sum_{k, l \in I}\left\langle x_{i}, h_{l(1)}\right\rangle\left\langle x_{j}, h_{l(2)} h_{k}\right\rangle \pi\left(x_{l} \otimes x_{k}\right) .
\end{aligned}
$$

This is easy to deduce that if $\pi=\mathrm{ev}_{a}$ as in (2.4) then

$$
\pi \mathcal{F}(x \otimes y)=\pi^{\prime}(x \otimes y), \quad \pi^{\prime}=\mathrm{ev}_{a^{\prime}}, \quad a^{\prime}=\left(\mathcal{F}^{\prime} \otimes \mathrm{id}_{V}\right)(a) \in H \otimes H \otimes V .
$$

Since $\mathcal{F}^{\prime}: H \otimes H \rightarrow H \otimes H$ is invertible, $a=0$ iff $a^{\prime}=0$. Hence, $\pi^{\prime}=\pi \mathcal{F}=0$ implies $\pi=0$.

For $\pi \mathcal{F}^{-1}$ the proof is completely analogous. 


\subsection{Pseudoalgebras}

In the exposition of the notion of pseudoalgebra we will preferably follow [1].

Hereinafter, $H$ is a cocommutative Hopf algebra, e.g., $H=U(\mathfrak{g})$ or $H=U(\mathfrak{g}) \# \mathbb{k}[\Gamma]$.

Definition 2 ([1]). Let $P$ be a left $H$-module. A pseudoproduct is an $H$-bilinear map

$$
*: P \otimes P \rightarrow(H \otimes H) \otimes_{H} P \text {. }
$$

An $H$-module $P$ endowed with a pseudoproduct $*$ is called a pseudoalgebra over $H$ (or $H$ pseudoalgebra). If $P$ is a finitely generated $H$-module, then $P$ is said to be finite pseudoalgebra.

For every $n, m \geq 1$, an $H$-bilinear map $*: P \otimes P \rightarrow(H \otimes H) \otimes_{H} P$ can be naturally expanded to a map from $\left(H^{\otimes n} \otimes_{H} P\right) \otimes\left(H^{\otimes m} \otimes_{H} P\right)$ to $\left(H^{\otimes n+m} \otimes_{H} P\right)$ :

$$
\left(F \otimes_{H} a\right) *\left(G \otimes_{H} b\right)=\left((F \otimes G) \otimes_{H} 1\right)\left(\left(\Delta^{[n]} \otimes \Delta^{[m]}\right) \otimes_{H} \operatorname{id}_{P}\right)(a * b),
$$

where $F \in H^{\otimes n}, G \in H^{\otimes m}, a, b \in P$.

This operation allows to consider long terms in $P$ with respect to $*$.

One of the main features of a cocommutative bialgebra $H$ is that symmetric groups $S_{n}$ act by $H$-module automorphisms on $H^{\otimes n}$ with respect to (2.1). The action of $\sigma \in S_{n}$ is defined by

$$
\sigma\left(h_{1} \otimes \cdots \otimes h_{n}\right)=h_{1 \sigma^{-1}} \otimes \cdots \otimes h_{n \sigma^{-1}} .
$$

Let us write down the obvious rules matching the action of $S_{n}$ with the "expanded" pseudoproduct (2.5). For every $A \in H^{\otimes n} \otimes_{H} P, B \in H^{\otimes m} \otimes_{H} P, \tau \in S_{n}, \sigma \in S_{m}$ we have

$$
\left(\left(\tau \otimes_{H} \operatorname{id}_{P}\right)(A)\right) * B=\left(\bar{\tau} \otimes_{H} \operatorname{id}_{P}\right)(A * B),
$$

where $\bar{\tau} \in S_{n+m}, k \bar{\tau}=k \tau$ for $k=1, \ldots, n, k \bar{\tau}=k$ for $k=n+1, \ldots, n+m$;

$$
A *\left(\left(\sigma \otimes_{H} \operatorname{id}_{P}\right)(B)\right)=\left(\sigma_{+n} \otimes_{H} \operatorname{id}_{P}\right)(A * B),
$$

where $i \sigma_{+n}=i$ for $i=1, \ldots, n,(n+j) \sigma_{+n}=n+j \sigma$ for $j=1, \ldots, m$.

A pseudoproduct $*: P \otimes P \rightarrow(H \otimes H) \otimes_{H} P$ can be completely described by a family of binary algebraic operations. Let $P$ be an $H$-pseudoalgebra, $X=H^{*}$. Lemma 1 implies that for every $a, b \in P$ their pseudoproduct has a unique presentation of the form

$$
a * b=\sum_{i}\left(h_{i} \otimes 1\right) \otimes_{H} c_{i},
$$

where $\left\{h_{i} \mid i \in I\right\}$ is a fixed basis of $H$. Consider the projections (called Fourier coefficients of $a * b)$

$$
\left(a \circ_{x} b\right)=\sum_{i}\left\langle x, S\left(h_{i}\right)\right\rangle c_{i} \in P,
$$

for all $x \in X$. The $x$-products obtained have the following properties:

locality

$$
\left(a \circ_{x_{i}} b\right)=0 \text { for almost all } i \in I
$$

sesqui-linearity

$$
\left(h a \circ_{x} b\right)=\left(a \circ_{x h} b\right), \quad\left(a \circ_{x} h b\right)=h_{(2)}\left(a_{S\left(h_{(1)}\right) x} b\right) .
$$

Note that the locality property does not depend on the choice of a basis in $H$ : (2.8) means that $\operatorname{codim}\left\{x \in X \mid\left(a \circ_{x} b\right)=0\right\}<\infty$. 
Remark 1 ([1]). In the case $H=\mathbb{k}[D], X \simeq \mathbb{k}[[t]]$, where $\left\langle t^{n}, D^{m}\right\rangle=n ! \delta_{n, m}$, the correspondence between conformal $n$-products $(n \geq 0)$ and the pseudoproduct is provided by

$$
a * b=\sum_{n \geq 0} \frac{1}{n !}\left((-D)^{n} \otimes 1\right) \otimes_{H}\left(a \circ_{n} b\right),
$$

i.e., $a \circ_{n} b=a \circ_{t^{n}} b, n \geq 0$.

In the same way, one may define Fourier coefficients of an arbitrary element $A \in H^{\otimes n} \otimes_{H} P$, $n \geq 2$. By Lemma $1 A$ can be uniquely presented as $A=\sum_{\bar{\imath}}\left(h_{i_{1}} \otimes \cdots \otimes h_{i_{n-1}} \otimes 1\right) \otimes_{H} a_{\bar{\imath}}$, $\bar{\imath}=\left(i_{1}, \ldots, i_{n-1}\right) \in I^{n-1}$. By abuse of terminology, we will call these $a_{\bar{\imath}} \in P$ Fourier coefficients of $A$.

There is a canonical way to associate an ordinary algebra $\mathcal{A}(P)$ with a given pseudoalgebra $P[1]$. As a linear space, $\mathcal{A}(P)$ coincides with $X \otimes_{H} P$, and the product is given by

$$
\left(x \otimes_{H} a\right)\left(y \otimes_{H} b\right)=S^{*}\left(x_{(1)}\right) y \otimes_{H}\left(a \circ_{x_{(2)}} b\right), \quad x, y \in X, \quad a, b \in P .
$$

The algebra $\mathcal{A}(P)$ obtained is called the annihilation algebra of $P$. If $P$ is a torsion-free $H$ module then the structure of $P$ can be reconstructed from $\mathcal{A}(P)$ [1].

In the case of conformal algebras $(H=\mathbb{k}[D])$, there is a slightly different construction called coefficient algebra [7, 8, 15]. Suppose $C$ is a conformal algebra and consider the space

Coeff $C=\mathbb{k}\left[t, t^{-1}\right] \otimes_{\mathbb{k}[D]} C$, where and $D$ acts on $\mathbb{k}\left[t, t^{-1}\right]$ as $t^{n} D=-n t^{n-1}$. Denote $t^{n} \otimes_{\mathbb{k}[D]} a$ by $a(n), a \in C, n \in \mathbb{Z}$. The product on Coeff $C$ is provided by

$$
a(n) b(m)=\sum_{s \geq 0}\left(\begin{array}{l}
n \\
s
\end{array}\right)\left(a \circ_{s} b\right)(n+m-s), \quad n, m \in \mathbb{Z}, \quad a, b \in C .
$$

An arbitrary conformal algebra can be embedded into a conformal algebra of formal power series over its coefficient algebra [7].

\subsection{Varieties of pseudoalgebras}

Suppose $\Omega$ is a variety of ordinary algebras over a field of zero characteristic. Then $\Omega$ is defined by a family of homogeneous polylinear identities. Such an identity can be written as

$$
\sum_{\sigma \in S_{n}} t_{\sigma}\left(x_{1 \sigma}, \ldots, x_{n \sigma}\right)=0
$$

where each $t_{\sigma}\left(y_{1}, \ldots, y_{n}\right)$ is a linear combination of non-associative words obtained from $y_{1} \ldots y_{n}$ by some bracketing.

Definition 3 ([13]). Let $\Omega$ be a variety of ordinary algebras defined by a family of homogeneous polylinear identities of the form (2.10). Then set the $\Omega$ variety of pseudoalgebras as the class of pseudoalgebras satisfying the respective "pseudo"-identities of the form

$$
\sum_{\sigma \in S_{n}}\left(\sigma \otimes_{H} \operatorname{id}_{C}\right) t_{\sigma}^{*}\left(x_{1 \sigma}, \ldots, x_{n \sigma}\right)=0
$$

where $t_{\sigma}^{*}$ means the same term $t_{\sigma}$ with respect to the pseudoproduct operation $*$.

If $P$ is an $\Omega$ pseudoalgebra (or, in particular, conformal algebra) then its annihilation (coefficient) algebra belongs to the $\Omega$ variety of ordinary algebras [13]. The converse is also true for conformal algebras. 
However, the class of $\Omega$ pseudoalgebras is not a variety in the ordinary sense: This class is not closed under Cartesian products.

The main object of our study is the class of Jordan pseudoalgebras. Recall that the variety of Jordan algebras is defined by the following identities:

$$
a b=b a, \quad((a a) b) a=(a a)(b a) .
$$

In the polylinear form (if char $\mathbb{k} \neq 2,3$ ) the second identity of $(2.12)$ can be rewritten as follows (see, e.g., [20]):

$$
[a b c d]+[d b c a]+[c b a d]=\{a b c d\}+\{a c b d\}+\{a d c b\} .
$$

Here $[\ldots]$ and $\{\ldots\}$ stand for the following bracketing schemes: $\left[a_{1} \ldots a_{n}\right]=\left(a_{1}\left[a_{2} \ldots a_{n}\right]\right)$, $\left\{a_{1} a_{2} a_{3} a_{4}\right\}=\left(\left(a_{1} a_{2}\right)\left(a_{3} a_{4}\right)\right)$.

Therefore, an $H$-module $P$ (over a cocommutative Hopf algebra $H$ ) endowed with a pseudoproduct $\circ$ is a Jordan pseudoalgebra iff it satisfies the following identities of the form (2.11):

$$
\begin{aligned}
& a \circ b=\left(\sigma_{12} \otimes_{H} \operatorname{id}_{P}\right)(b \circ a), \\
& {[a \circ b \circ c \circ d]+\left(\sigma_{14} \otimes_{H} \operatorname{id}_{P}\right)[d \circ b \circ c \circ a]+\left(\sigma_{13} \otimes_{H} \operatorname{id}_{P}\right)[c \circ b \circ a \circ d]} \\
& \quad=\{a \circ b \circ c \circ d\}+\left(\sigma_{23} \otimes_{H} \operatorname{id}_{P}\right)\{a \circ c \circ b \circ d\}+\left(\sigma_{24} \otimes_{H} \operatorname{id}_{P}\right)\{a \circ d \circ c \circ b\},
\end{aligned}
$$

where $\sigma_{i j}=(i j)$ are the transpositions from $S_{4}$.

As in the case of ordinary algebras, the natural relations hold between associative, Lie, and Jordan pseudoalgebras. An associative pseudoalgebra $P$ with respect to the new pseudoproduct

$$
[a * b]=a * b-\left(\sigma_{12} \otimes_{H} \operatorname{id}_{P}\right)(b * a)
$$

is a Lie pseudoalgebra denoted by $P^{(-)}$[1]. Similarly, another pseudoproduct $\circ$ given by

$$
a \circ b=a * b+\left(\sigma_{12} \otimes_{H} \operatorname{id}_{P}\right)(b * a)
$$

makes $P$ into a Jordan pseudoalgebra $P^{(+)}[13]$.

Example 1. Let $H^{\prime}$ be a Hopf subalgebra of $H$, and let $P^{\prime}$ be an $H^{\prime}$-pseudoalgebra with respect to a pseudoproduct $*^{\prime}$. Define a pseudoproduct on $P=H \otimes_{H^{\prime}} P^{\prime}$ by linearity:

$$
\left(h \otimes_{H^{\prime}} a\right) *\left(g \otimes_{H^{\prime}} b\right)=\sum_{i}\left(h h_{i} \otimes g g_{i}\right) \otimes_{H}\left(1 \otimes_{H^{\prime}} c_{i}\right), \quad g, h \in H,
$$

where $a *^{\prime} b=\sum_{i}\left(h_{i} \otimes g_{i}\right) \otimes_{H^{\prime}} c_{i}, a, b, c_{i} \in P^{\prime}$. The pseudoalgebra $P$ obtained is called the current pseudoalgebra $\operatorname{Cur}_{H^{\prime}}^{H} P^{\prime}$.

In particular, $\mathbb{k} \subset H$ is a Hopf subalgebra of $H$. Hence, an ordinary algebra $A$ gives rise to current pseudoalgebra $\operatorname{Cur} A=\operatorname{Cur}_{\mathbb{k}}^{H} A$.

It is clear that if $P^{\prime}$ is an $\Omega$ pseudoalgebra over $H^{\prime}$ then so is $\operatorname{Cur}_{H^{\prime}}^{H} P^{\prime}$.

Example 2. Consider $H=U(\mathfrak{h})$, where $\mathfrak{h}$ is a Lie algebra. Then the free left $H$-module $H \otimes H$ equipped by pseudoproduct

$$
(h \otimes a) *(g \otimes b)=\left(h b_{(1)} \otimes g\right) \otimes_{H}\left(1 \otimes a b_{(2)}\right), \quad a, b, g, h \in H,
$$

is an associative pseudoalgebra. The submodule $W(\mathfrak{h})=H \otimes \mathfrak{h}$ is a subalgebra of the corresponding Lie pseudoalgebra $(H \otimes H)^{(-)}$.

Note that if $\mathfrak{h}^{\prime}$ is a Lie subalgebra of $\mathfrak{h}$, then $H^{\prime}=U\left(\mathfrak{h}^{\prime}\right)$ is a Hopf subalgebra of $H$, and $\operatorname{Cur}_{H^{\prime}}^{H} W\left(\mathfrak{h}^{\prime}\right)$ is actually a subalgebra of $W(\mathfrak{h})$. 
In particular, if $\mathfrak{h}$ is the 1-dimensional Lie algebra then $W(\mathfrak{h})$ is just the Virasoro conformal algebra [7].

Later we will use the classification of simple finite Lie pseudoalgebras [1]. Although the results obtained in [1] are much more explicit, the following statements are sufficient for our purposes.

Theorem 1 ([1]). A simple finite Lie pseudoalgebra $L$ over $H=U(\mathfrak{h})$, $\operatorname{dim} \mathfrak{h}<\infty, \mathbb{k}=\mathbb{C}$, is isomorphic either to Cur $\mathfrak{g}$, where $\mathfrak{g}$ is a simple finite-dimensional Lie algebra, or to a subalgebra of $W(\mathfrak{h})$.

Theorem 2 ([1]). A simple Lie pseudoalgebra $L$ over $H=U(\mathfrak{h}) \# \mathbb{k}[\Gamma]$ which is finite over $U(\mathfrak{h})$ $(\operatorname{dim} \mathfrak{h}<\infty, \mathbb{k}=\mathbb{C})$ is a finite direct sum of isomorphic simple $U(\mathfrak{h})$-pseudoalgebras such that $\Gamma$ acts on them transitively.

\subsection{Conformal linear maps}

Let $H$ be a cocommutative Hopf algebra, and let $M_{1}, M_{2}$ be two left $H$-modules. A map $\varphi: M_{1} \rightarrow(H \otimes H) \otimes_{H} M_{2}$ is said to be (left) conformal linear if

$$
\varphi(h a)=(1 \otimes h) \varphi(a), \quad h \in H, \quad a \in M_{1} .
$$

The set of all left conformal linear maps is denoted by $\operatorname{Chom}^{l}\left(M_{1}, M_{2}\right)$. For $M_{1}=M_{2}=M$ we denote $\operatorname{Chom}^{l}(M, M)=\operatorname{Cend}^{l}(M)$.

For every $H$-modules $M_{1}, M_{2}$, the set $\operatorname{Chom}^{l}\left(M_{1}, M_{2}\right)$ can be considered as an $H$-module with respect to the action

$$
h \varphi(a)=(h \otimes 1) \varphi(a), \quad h \in H, \quad \varphi \in \operatorname{Chom}^{l}\left(M_{1}, M_{2}\right), \quad a \in M_{1} .
$$

For example, if $P$ is a pseudoalgebra, $a \in P$, then the operator of left multiplication $L_{a}: b \mapsto$ $a * b, b \in P$, belongs to $\operatorname{Cend}^{l}(P)$.

In order to unify notations, we will use $\varphi * a$ for $\varphi(a), a \in M, \varphi \in \operatorname{Cend}^{l}(M)$. One may consider $*$ here as an $H$-bilinear map from $\operatorname{Cend}^{l}(M) \otimes M$ to $(H \otimes H) \otimes_{H} M$. The relation (2.5) allows to expand this map to

$$
\left(H^{\otimes n} \otimes_{H} \operatorname{Cend}^{l}(M)\right) \otimes\left(H^{\otimes m} \otimes_{H} M\right) \rightarrow H^{\otimes n+m} \otimes_{H} M .
$$

The correspondence between $\varphi * a$ and $\left(\varphi \circ_{x} a\right)(x \in X)$ is given by

$$
\varphi * a=\sum_{i \in I}\left(S\left(h_{i}\right) \otimes 1\right) \otimes_{H}\left(\varphi \circ_{x_{i}} a\right) .
$$

The space $\operatorname{Cend}^{l}(M)$ can be also endowed with a family of $x$-products given by

$$
\left(\varphi \circ_{x} \psi\right) \circ_{y} a=\left(\varphi \circ_{x_{(2)}}\left(\psi \circ_{S^{*}\left(x_{(1)}\right) y} a\right)\right), \quad \varphi, \psi \in \operatorname{Cend}^{l}(M), \quad x, y \in X,
$$

for $a \in M$.

The $x$-products $\left(\cdot \circ_{x} \cdot\right)$ on $\operatorname{Cend}^{l}(M)$ satisfy (2.9), but (2.8) does not hold, in general. To ensure the locality, it is sufficient to assume that $M$ is a finitely generated $H$-module [1, Section 10]. Therefore, $\operatorname{Cend}^{l}(M)$ for a finitely generated $H$-module $M$ is an associative $H$ pseudoalgebra.

For a finite pseudoalgebra $P$, it is easy to rewrite the identity (2.13) using the operators of left multiplication. Namely, this identity is equivalent to

$$
\begin{aligned}
& L_{a} * L_{b} * L_{c}+\left(\sigma_{13} \otimes_{H} \mathrm{id}\right)\left(L_{c} * L_{b} * L_{a}\right)+\left(\sigma_{123} \otimes_{H} \mathrm{id}\right) L_{b *(c * a)} \\
& \quad=L_{a * b} * L_{c}+\left(\sigma_{23} \otimes_{H} \mathrm{id}\right)\left(L_{a * c} * L_{b}\right)+\left(\sigma_{13} \otimes_{H} \mathrm{id}\right)\left(L_{c * b} * L_{a}\right),
\end{aligned}
$$

where $\sigma_{123}$ denotes the permutation $(123) \in S_{4}$. 


\section{Tits-Kantor-Koecher construction for finite Jordan pseudoalgebras}

The general scheme described in $[10,11,12,18]$ provides an embedding of a Jordan algebra into a Lie algebra. It is called the Tits-Kantor-Koecher (TKK) construction for Jordan algebras.

Let us recall the TKK construction for ordinary algebras. For a Jordan algebra $\mathfrak{j}$, the set of derivations $\operatorname{Der}(\mathfrak{j})$ is a Lie subalgebra of $\operatorname{End}(\mathfrak{j})$ with respect to the commutator of linear maps. Consider the (formal) direct $\operatorname{sum} \mathrm{S}(\mathfrak{j})=\operatorname{Der}(\mathfrak{j}) \oplus L(\mathfrak{j})$, where $L(\mathfrak{j})$ is the linear space of all left multiplications $L_{a}: b \mapsto a b, a \in \mathfrak{j}$. It is well-known that $[L(\mathfrak{j}), L(\mathfrak{j})] \subseteq \operatorname{Der}(\mathfrak{j})$. Then the space $S(\mathfrak{j})$ with respect to the new operation $[\cdot, \cdot]$ given by

$$
\left[\left(L_{a}+D\right),\left(L_{b}+T\right)\right]=L_{D b}-L_{T a}+\left[L_{a}, L_{b}\right]+[D, T] .
$$

is a Lie algebra called the structure Lie algebra of $\mathfrak{j}$. Finally, consider

$$
T(\mathfrak{j})=\mathfrak{j}^{-} \oplus \mathrm{S}_{0}(\mathfrak{j}) \oplus \mathfrak{j}^{+},
$$

where $\mathfrak{j}^{ \pm} \simeq \mathfrak{j}, \mathrm{S}_{0}(\mathfrak{j})$ is the subalgebra of $\mathrm{S}(\mathfrak{j})$, generated by $U_{a, b}=L_{a b}+\left[L_{a}, L_{b}\right] \in \mathrm{S}(\mathfrak{j}), a, b \in \mathfrak{j}$. Let us endow $\mathrm{T}(\mathfrak{j})$ with the following operation:

$$
\begin{aligned}
& {\left[\Sigma, a^{-}\right]=(\Sigma a)^{-}, \quad\left[a^{-}, b^{+}\right]=U_{a, b},} \\
& {\left[a^{+}, b^{+}\right]=\left[a^{-}, b^{-}\right]=0, \quad\left[a^{-}, \Sigma\right]=-(\Sigma a)^{-}, \quad\left[a^{+}, \Sigma\right]=-\left(\Sigma^{*} a\right)^{+},} \\
& {\left[\Sigma, a^{+}\right]=\left(\Sigma^{*} a\right)^{+}, \quad\left[a^{+}, b^{-}\right]=U_{a, b}^{*},}
\end{aligned}
$$

where $\Sigma^{*}=-L_{a}+D$ for $\Sigma=L_{a}+D \in \mathrm{S}(\mathfrak{j})$. This operation makes $\mathrm{T}(\mathfrak{j})$ to be a Lie algebra called the TKK construction for $\mathfrak{j}$.

In the case of conformal algebras, a similar construction was introduced in [19] by making use of coefficient algebras. We are going to get an analogue of TKK construction for finite Jordan pseudoalgebras using the language of pseudoalgebras rather than annihilation algebras.

Definition 4. Let $P$ be an $H$-pseudoalgebra. A conformal endomorphism $T \in \operatorname{Cend}^{l}(P)$ is said to be a (left) pseudoderivation, if

$$
T *(a * b)=(T * a) * b+\left(\sigma_{12} \otimes_{H} \operatorname{id}_{P}\right)(a *(T * b))
$$

for all $a, b \in P$. The set of all pseudoderivations of $P$ we denote by $\operatorname{Der}^{l}(P)$.

In particular, if $P$ is a finite pseudoalgebra then (3.1) is equivalent to $\left[T * L_{a}\right]=L_{T * a}, a \in P$.

Lemma 3. Suppose that for some $A \in(H \otimes H) \otimes_{H} \operatorname{Cend}^{l}(P)$ the equality

$$
A *(a * b)=(A * a) * b+\left(\sigma_{132} \otimes_{H} \mathrm{id}\right)(a *(A * b))
$$

holds for all $a, b \in P$. Then all Fourier coefficients of $A$ belong to in $\operatorname{Der}^{l}(P)$.

Proof. For every $B \in H^{\otimes n+1} \otimes_{H} M$ ( $M$ is an $H$-module), there exists a unique presentation

$$
B=\sum_{\bar{\imath}}\left(G_{\bar{\imath}} \otimes 1\right) \otimes_{H} b_{\bar{\imath}}
$$

where $G_{\bar{\imath}}$ form a linear basis of $H^{\otimes n}$ (see Lemma 1). By $B_{x_{1}, \ldots, x_{n}}, x_{i} \in X$, we denote the expression

$$
\sum_{\bar{\imath}}\left\langle x_{1} \otimes \cdots \otimes x_{n}, G_{\bar{\imath}}\right\rangle b_{\bar{\imath}} .
$$


It is clear that the map $\left(x_{1}, \ldots, x_{n}\right) \mapsto B_{x_{1}, \ldots, x_{n}}$ is polylinear. If we fix an arbitrary set of $n-2$ arguments, then the map $X \otimes X \rightarrow M$ obtained is local in the sense of Definition 1.

Let $A=\sum_{i \in I}\left(h_{i} \otimes 1\right) \otimes_{H} D_{i}, a * b=\sum_{j \in I}\left(h_{j} \otimes 1\right) \otimes_{H} c_{j}, D_{i} * c_{j}=\sum_{k \in I}\left(h_{k} \otimes 1\right) \otimes_{H} d_{i j k}$. Then

$$
\begin{aligned}
& D_{i} *(a * b)=\sum_{j, k \in I}\left(h_{k} \otimes h_{j} \otimes 1\right) \otimes_{H} d_{i j k}, \\
& A *(a * b)=\sum_{i, j, k \in I}\left(h_{i} h_{k(1)} \otimes h_{k(2)} \otimes h_{j} \otimes 1\right) \otimes_{H} d_{i j k} .
\end{aligned}
$$

Compare (3.2) and (3.3) to get

$$
(A *(a * b))_{x, y, z}=\sum_{i \in I}\left\langle x_{(1)}, h_{i}\right\rangle\left(D_{i} *(a * b)\right)_{x_{(2)} y, z}
$$

In the same way,

$$
\begin{aligned}
& ((A * a) * b)_{x, y, z}=\sum_{i \in I}\left\langle x_{(1)}, h_{i}\right\rangle\left(\left(D_{i} * a\right) * b\right)_{x_{(2)} y, z} \\
& \left(\left(\sigma_{132} \otimes_{H} \text { id }\right)(a *(A * b))\right)_{x, y, z}=\sum_{i \in I}\left\langle x_{(1)}, h_{i}\right\rangle\left(\left(\sigma_{12} \otimes_{H} \mathrm{id}\right)\left(a *\left(D_{i} * b\right)\right)\right)_{x_{(2)} y, z} .
\end{aligned}
$$

The relations (3.4)-(3.6) together with Lemma 2 imply

$$
\left.\pi(x, y, z)=\sum_{i \in I}\left\langle x, h_{i}\right\rangle\left(D_{i} *(a * b)-\left(D_{i} * a\right) * b\right)-\left(\sigma_{12} \otimes_{H} \mathrm{id}\right)\left(\left(D_{i} * a\right) * b\right)\right)_{y, z}=0
$$

It means that

$$
\left.D_{i} *(a * b)-\left(D_{i} * a\right) * b\right)-\left(\sigma_{12} \otimes_{H} \mathrm{id}\right)\left(a *\left(D_{i} * b\right)\right)=0 .
$$

Lemma 4. For a finite pseudoalgebra $P$ the set of all pseudoderivations is a subalgebra of the Lie pseudoalgebra $\operatorname{Cend}^{l}(P)^{(-)}$.

Proof. Let $D_{1}, D_{2} \in \operatorname{Der}^{l}(P)$, i.e., $\left[D_{i} * L_{a}\right]=L_{D_{i} * a}$ for $a \in P, i=1,2$.

Since $\operatorname{Cend}^{l}(P)^{(-)}$satisfies Jacobi identity,

$$
\begin{aligned}
{\left[\left[D_{1} * D_{2}\right] * L_{a}\right] } & =\left[D_{1} *\left[D_{2} * L_{a}\right]\right]-\left(\sigma_{12} \otimes_{H} \mathrm{id}\right)\left(\left[D_{2} *\left[D_{1} * L_{a}\right]\right]\right) \\
& =\left[D_{1} * L_{D_{2} * a}\right]-\left(\sigma_{12} \otimes_{H} \mathrm{id}\right)\left(\left[D_{2} * L_{D_{1} * a}\right]\right) \\
& =L_{D_{1} *\left(D_{2} * a\right)}-\left(\sigma_{12} \otimes_{H} \mathrm{id}\right) L_{D_{2} *\left(D_{1} * a\right)}=L_{\left[D_{1} * D_{2}\right] * a} .
\end{aligned}
$$

Hence, for every $a, b \in P$ we have

$$
\left[D_{1} * D_{2}\right] *(a * b)=\left(\left[D_{1} * D_{2}\right] * a\right) * b+\left(\sigma_{132} \otimes_{H} \mathrm{id}\right)\left(a *\left(\left[D_{1} * D_{2}\right] * b\right)\right) .
$$

Lemma 3 implies that $\left[D_{1} \circ_{x} D_{2}\right] \in \operatorname{Der}^{l}(P)$ for all $x \in X$.

Lemma 5. Let $J$ be a finite Jordan pseudoalgebra, and let $L(J)$ be the $H$-submodule of $\mathrm{Cend}^{l}(J)$ generated by $\left\{L_{a} \mid a \in J\right\}$. Then $\left[\begin{array}{lll}L_{a} & \circ_{x} & L_{b}\end{array}\right]$ is a pseudoderivation for every $x \in X$, i.e., $L^{\prime}(J) \subseteq \operatorname{Der}^{l}(J)$ 
Proof. The following relation is easy to deduce from (2.13):

$$
\begin{aligned}
& L_{a} * L_{c * d}+\left(\sigma_{13} \otimes_{H} \mathrm{id}\right)\left(L_{d} * L_{c * a}\right)+\left(\sigma_{12} \otimes_{H} \mathrm{id}\right)\left(L_{c} * L_{a * d}\right) \\
& \quad=\left(\sigma_{123} \otimes_{H} \mathrm{id}\right)\left(L_{c * d} * L_{a}\right)+L_{a * c} * L_{d}+\left(\sigma_{23} \otimes_{H} \mathrm{id}\right)\left(L_{a * d} * L_{c}\right) .
\end{aligned}
$$

So by $(2.6),(2.7)$

$$
\left[L_{a} * L_{c * d}\right]=\left[L_{a * c} * L_{d}\right]-\left(\sigma_{12} \otimes_{H} \mathrm{id}\right)\left[L_{c} * L_{a * d}\right] .
$$

It is sufficient to prove that for every $a, b, c \in J$ we have $\left[\left[L_{a} * L_{b}\right] * L_{c}\right]=L_{\left[L_{a} * L_{b}\right] * c}$, i.e.,

$$
\begin{gathered}
L_{a} * L_{b} * L_{c}-\left(\sigma_{132} \otimes_{H} \mathrm{id}\right)\left(L_{c} * L_{a} * L_{b}\right)+\left(\sigma_{13} \otimes_{H} \mathrm{id}\right) L_{c} * L_{b} * L_{a} \\
-\left(\sigma_{12} \otimes_{H} \mathrm{id}\right) L_{b} * L_{a} * L_{c}=L_{a *(b * c)}-\left(\sigma_{12} \otimes_{H} \mathrm{id}\right) L_{b *(a * c)} .
\end{gathered}
$$

Indeed,

$$
\begin{array}{rl}
L_{a} * L_{b} & * L_{c}+\left(\sigma_{13} \otimes_{H} \mathrm{id}\right)\left(L_{c} * L_{b} * L_{a}\right)=-\left(\sigma_{123} \otimes_{H} \mathrm{id}\right) L_{b *(c * a)} \\
& +L_{a * b} * L_{c}+\left(\sigma_{23} \otimes_{H} \mathrm{id}\right)\left(L_{a * c} * L_{b}\right)+\left(\sigma_{13} \otimes_{H} \mathrm{id}\right)\left(L_{c * b} * L_{a}\right), \\
\left(\sigma_{12} \otimes_{H} \mathrm{id}\right)\left(L_{b} * L_{a} * L_{c}\right)+\left(\sigma_{132} \otimes_{H} \mathrm{id}\right)\left(L_{c} * L_{a} * L_{b}\right) & \\
= & \left(\sigma_{12} \otimes_{H} \mathrm{id}\right)\left(L_{b} * L_{a} * L_{c}\right)+\left(\sigma_{12} \sigma_{13} \otimes_{H} \mathrm{id}\right)\left(L_{c} * L_{a} * L_{b}\right) \\
= & -\left(\sigma_{12} \sigma_{123} \otimes_{H} \mathrm{id}\right) L_{a *(c * b)}+\left(\sigma_{12} \otimes_{H} \mathrm{id}\right)\left(L_{b * a} * L_{c}\right) \\
& +\left(\sigma_{12} \sigma_{23} \otimes_{H} \mathrm{id}\right)\left(L_{b * c} * L_{a}\right)+\left(\sigma_{12} \sigma_{13} \otimes_{H} \mathrm{id}\right)\left(L_{c * a} * L_{b}\right) \\
= & -\left(\sigma_{23} \otimes_{H} \mathrm{id}\right) L_{a *(c * b)}+\left(\sigma_{12} \otimes_{H} \mathrm{id}\right)\left(L_{b * a} * L_{c}\right) \\
& +\left(\sigma_{123} \otimes_{H} \mathrm{id}\right)\left(L_{b * c} * L_{a}\right)+\left(\sigma_{132} \otimes_{H} \mathrm{id}\right)\left(L_{c * a} * L_{b}\right) .
\end{array}
$$

Subtracting (3.9) from (3.10) and using commutativity $L_{a * b}=\left(\sigma_{12} \otimes_{H}\right.$ id $) L_{b * a}$, we obtain (3.8).

Definition 5. Let $J$ be a finite Jordan pseudoalgebra. The formal direct sum of $H$-modules

$$
\mathrm{S}(J)=L(J) \oplus \operatorname{Der}^{l}(J)
$$

endowed with the pseudoproduct

$$
\left[\left(L_{a}+D\right) *\left(L_{b}+T\right)\right]=L_{D * b}-\left(\sigma_{12} \otimes_{H} \text { id }\right) L_{T * a}+\left[L_{a} * L_{b}\right]+[D * T]
$$

is called the structure Lie pseudoalgebra of $J$.

It is straightforward to check that the (pseudo) anticommutativity and Jacobi identities hold for $(3.11)$.

Consider the elements $U_{a, b}=L_{a * b}+\left[L_{a} * L_{b}\right] \in(H \otimes H) \otimes_{H} \mathrm{~S}(J), a, b \in J$. By $U_{\left(a \circ_{x} b\right)}=$ $L_{\left(a \circ_{x} b\right)}+\left[L_{a} \circ_{x} L_{b}\right], x \in X$, we denote the Fourier coefficients of $U_{a, b}$. The linear space $\mathrm{S}_{0}(J)$ generated by the set $\left\{U_{(a \circ x)} \mid a, b \in J, x \in X\right\}$ is an $H$-submodule of $\mathrm{S}(J)$.

Proposition 1. The $H$-module $\mathrm{S}_{0}(J)$ is closed under the pseudoproduct (3.11), i.e., $\mathrm{S}_{0}(J)$ is a Lie pseudoalgebra.

Proof. Let us calculate $\left[U_{a, b} * U_{c, d}\right], a, b, c, d \in J$. Denote $D=\left[L_{a} * L_{b}\right], A=a * b$. Then $\left[U_{a, b} * L_{c * d}\right]=\left[L_{A} * L_{c * d}\right]+L_{(D * c) * d}+\left(\sigma_{132} \otimes_{H}\right.$ id $) L_{c *(D * d)},\left[U_{a, b} *\left[L_{c} * L_{d}\right]\right]=\left[L_{A} *\left[L_{c} * L_{d}\right]\right]+$ $\left[L_{D * c} * L_{d}\right]+\left(\sigma_{132} \otimes_{H}\right.$ id $)\left[L_{c} * L_{D * d}\right]$. Therefore,

$$
\left[U_{a, b} * U_{c, d}\right]=\left[L_{A} * L_{c * d}\right]+\left[L_{A} *\left[L_{c} * L_{d}\right]\right]+U_{D * c, d}+\left(\sigma_{132} \otimes_{H} \mathrm{id}\right) U_{c, D * d} .
$$

From the first summand of the right-hand side we obtain $\left[L_{A} * L_{c * d}\right]=\left[L_{A * c} * L_{d}\right]-\left(\sigma_{132} \otimes_{H}\right.$ id) $\left[L_{c} * L_{A * d}\right]$ by using (3.7). Moreover, $\left[L_{A} *\left[L_{c} * L_{d}\right]\right]=L_{(A * c) * d}-\left(\sigma_{132} \otimes_{H}\right.$ id $) L_{c *(A * d)}$. Hence,

$$
\left[U_{a, b} * U_{c, d}\right]=U_{A * c, d}+U_{D * c, d}+\left(\sigma_{132} \otimes_{H} \mathrm{id}\right)\left(U_{c, D * d}-U_{c, A * d}\right) .
$$


Denote $U_{a, b}^{*}=-L_{a * b}+\left[L_{a} * L_{b}\right], a, b \in J$. Note that $U_{a, b}^{*}=-\left(\sigma_{12} \otimes_{H}\right.$ id $) U_{b, a}$, so all Fourier coefficients of $U_{a, b}^{*}$ lie in $\mathrm{S}_{0}(J)$. If $J$ is a Jordan pseudoalgebra and $J^{2}=J$, i.e., every $a \in J$ lies in the subspace generated by the set $\left\{\left(b \circ_{x} c\right) \mid b, c \in J, x \in X\right\}$, then $\mathrm{S}_{0}(J) \supset L(J)$.

Indeed, for every $a, b \in J$ we have $U_{a, b}+\left(\sigma_{12} \otimes_{H} \operatorname{id}_{J}\right) U_{b, a}=2 L_{a * b}$, so $\left.L_{(a \circ x} b\right) \in \mathrm{S}_{0}(J)$. Hence, $L(J)=L\left(J^{2}\right) \subset \mathrm{S}_{0}(J)$.

Let us consider the direct sum of $H$-modules

$$
\mathrm{T}(J)=J^{-} \oplus \mathrm{S}_{0}(J) \oplus J^{+},
$$

where $J^{+}$and $J^{-}$are isomorphic copies of $J$. Given $a \in J$ (or $A \in H^{\otimes n} \otimes_{H} J$ ), we will denote by $a^{ \pm}$(or $A^{ \pm}$) the image of this element in $J^{ \pm}$(or $H^{\otimes n} \otimes_{H} J^{ \pm}$). Define a pseudoproduct on $\mathrm{T}(J)$ by the following rule: for $a^{ \pm}, b^{ \pm} \in J^{ \pm}, \Sigma \in \mathrm{S}_{0}(J)$ set

$$
\begin{aligned}
& {\left[a^{+} * b^{-}\right]=U_{a, b}^{*}, \quad\left[a^{-} * b^{+}\right]=U_{a, b}, \quad\left[a^{+} * b^{+}\right]=\left[a^{-} * b^{-}\right]=0,} \\
& {\left[a^{-} * \Sigma\right]=-\left(\sigma_{12} \otimes_{H} \text { id }\right)(\Sigma * a)^{-}, \quad\left[\Sigma * a^{-}\right]=(\Sigma * a)^{-},} \\
& {\left[a^{+} * \Sigma\right]=-\left(\sigma_{12} \otimes_{H} \text { id }\right)\left(\Sigma^{*} * a\right)^{+}, \quad\left[\Sigma * a^{+}\right]=\left(\Sigma^{*} * a\right)^{+} .}
\end{aligned}
$$

Set the pseudoproduct on $\mathrm{S}_{0}(J)$ to be the same as (3.11). Here we have used $\Sigma^{*}=-L_{a}+D$ for $\Sigma=L_{a}+D \in \mathrm{S}(J)$.

Denote the projections of $\mathrm{T}(J)$ on $J^{+}, J^{-}, \mathrm{S}_{0}(J)$ by $\pi_{+}, \pi_{-}, \pi_{0}$, respectively. It is straightforward to check that $\mathrm{T}(J)$ is a Lie pseudoalgebra. This is an analogue of the Tits-Kantor-Koecher construction for an ordinary Jordan algebra.

Note that the structure pseudoalgebra is a formal direct sum of the corresponding $H$-modules, so the condition

$$
\Sigma * b=0 \quad \text { for all } \quad b \in J
$$

does not imply $\Sigma=0$ in $\mathrm{S}(J)$. However, if $\Sigma=L_{a}+D \in \mathrm{S}(J)$ and $\left[\Sigma * b^{-}\right]=\left[\Sigma * b^{+}\right]=0$ in $\mathrm{T}(J)$ for all $b \in J$, then $a * b+D * b=0$ and $-a * b+D * b=0$ by (3.12). Therefore, $a * b=D * b=0$ for all $b \in J$, i.e., $\Sigma=0$ in $\mathrm{S}(J)$.

Proposition 2. Let $J$ be a simple finite Jordan pseudoalgebra. Then $\mathcal{L}=\mathrm{T}(J)$ is a simple finite Lie pseudoalgebra.

Proof. Suppose that there exists a non-zero proper ideal $I \triangleleft \mathcal{L}$. Let

$$
J_{ \pm}=\left\{a \in J \mid a^{ \pm}=\pi_{ \pm}(b) \text { for some } b \in I\right\} .
$$

Since $J^{2}=J$, we have $\mathcal{L} \supset L(J)$. Hence, $J_{ \pm} \triangleleft J$.

Analogously, $J_{ \pm}^{0}=\left\{a \in J \mid a^{ \pm} \in I \cap J^{ \pm}\right\}$are also some ideals in $J$.

1) Consider the case $J_{+}=J_{-}=0$ (hence, $J_{+}^{0}=J_{-}^{0}=0$ ). Since $I \neq 0$, there exists $\Sigma=L_{b}+D \in \mathrm{S}_{0}(J) \cap I, \Sigma \neq 0$. But $\left[\Sigma * J^{ \pm}\right] \subseteq H^{\otimes 2} \otimes_{H} J_{ \pm}^{0}=0$, so $\Sigma=0$ as we have shown above, which is a contradiction.

2) Let $J_{+}=J, J_{-}^{0}=0$. Then for each $a \in J$ there exists $a^{+}+\Sigma+d^{-} \in I$. Consider

$$
\left[\left[\left(a^{+}+\Sigma+d^{-}\right) * b^{-}\right] * c^{-}\right]=\left[\left(U_{a, b}^{*}+(\Sigma * b)^{-}\right) * c^{-}\right]=\left(U_{a, b}^{*} * c\right)^{-} \in H^{\otimes 3} \otimes_{H} J_{-}^{0}=0 .
$$

For every $a, b, c \in J$ we have

$$
-L_{a * b} * c+\left[L_{a} * L_{b}\right] * c=0 .
$$

If $a * b=\sum_{i}\left(h_{i} \otimes 1\right) \otimes_{H}\left(a \circ_{x_{i}} b\right)$, then $b * a=\sum_{i}\left(1 \otimes h_{i}\right) \otimes_{H}\left(a \circ_{x_{i}} b\right)$ by commutativity. Therefore, $L_{a * b}=\sum_{i}\left(h_{i} \otimes 1\right) \otimes_{H} L_{a \circ_{x_{i}} b}=\left(\sigma_{12} \otimes_{H} \operatorname{id}_{L(J)}\right) L_{b * a}$. By the definition of commutator, 
$\left(\sigma_{12} \otimes_{H} \operatorname{id}_{J}\right)\left(\left[L_{a} * L_{b}\right] * c\right)=-\left[L_{b} * L_{a}\right] * c$. Relation (3.13) implies $-L_{b * a} * c+\left[L_{b} * L_{a}\right] * c=0$ by symmetry. Hence, $0=\left(\sigma_{12} \otimes_{H} \operatorname{id}_{J}\right)\left(-L_{b * a} * c+\left[L_{b} * L_{a}\right] * c\right)=-L_{a * b} * c-\left[L_{a} * L_{b}\right] * c$. Compare the last relation with (3.13) to get $L_{a * b} * c=0$ for all $a, b, c \in J$. Then the condition $J^{2}=J$ implies $L(J)=0$, which is a contradiction.

3) The case $J_{-}=J, J_{+}^{0}=0$ is completely analogous.

Hereby, if either of the ideals $J_{ \pm}^{0}$ is zero, then at least one of the ideals $J_{ \pm} \triangleleft J$ has to be zero, which is impossible. Hence, $J_{+}^{0}=J_{-}^{0}=J$, i.e., $I \supset J^{+}, J^{-}$. Since the whole pseudoalgebra $\mathcal{L}$ is generated by $J^{+} \cup J^{-}$, we have $I=\mathcal{L}$.

\section{Structure of simple Jordan pseudoalgebras}

We have shown (Proposition 2), that for a simple finite Jordan pseudoalgebra $J$ its TKK construction $\mathcal{L}=\mathrm{T}(J)$ is a simple finite Lie pseudoalgebra. This allows to describe simple Jordan pseudoalgebras using the classification of simple Lie pseudoalgebras [1].

\subsection{The case $H=U(\mathfrak{h})$}

Throughout this subsection, $H$ is the universal enveloping Hopf algebra of a finite-dimensional Lie algebra $\mathfrak{h}$ over $\mathbb{C}$.

Proposition 3. Let $J$ be a simple finite Jordan H-pseudoalgebra. Then the TKK construction $\mathrm{T}(J)$ is isomorphic to the current algebra Cur $\mathfrak{g}$ over a simple finite-dimensional Lie algebra $\mathfrak{g}$.

Proof. If $J$ is a simple finite Jordan $H$-pseudoalgebra, then $\mathrm{T}(J)$ is a simple finite Lie pseudoalgebra. Hence, either $\mathrm{T}(J)=$ Cur $\mathfrak{g}$, where $\mathfrak{g}$ is a simple finite-dimensional Lie algebra, or $\mathrm{T}(J)$ is a subalgebra in $W(\mathfrak{h})$ (see Theorem 1 and Example 2). The second case could not be realized since by [1, Proposition 13.6] the pseudoalgebra $W(\mathfrak{h})$ does not contain abelian subalgebras. This is not the case for $\mathrm{T}(J)$.

It remains to show that if $\mathrm{T}(J)=J^{+} \oplus \mathrm{S}_{0}(J) \oplus J^{-}=$Cur $\mathfrak{g}$ then $J$ is the current pseudoalgebra over a simple finite-dimensional Jordan algebra.

Suppose $e_{1}, \ldots, e_{n}$ is a basis of $\mathfrak{h}$. Then the set of monomials

$$
e^{(\alpha)}=e_{1}^{\left(\alpha_{1}\right)} \ldots e_{n}^{\left(\alpha_{n}\right)}, \quad \alpha=\left(\alpha_{1}, \ldots, \alpha_{n}\right) \in \mathbb{Z}^{n}, \quad \alpha_{i} \geq 0,
$$

where $e_{i}^{\left(\alpha_{i}\right)}=\frac{1}{\alpha_{i} !} e^{\alpha_{i}}$, is a basis of $H$. In order to simplify notation, we assume $e^{(\alpha)}=0$ whenever $\alpha$ contains a negative component.

Denote $|\alpha|=\alpha_{1}+\cdots+\alpha_{n}$. We will use the standard deg-lex order on the set of monomials of the form $e^{(\alpha)}: e^{(\alpha)} \leq e^{(\beta)}$ if and only if $\alpha \leq \beta$, i.e., either $|\alpha|<|\beta|$ or $|\alpha|=|\beta|$ and $\alpha$ is lexicographically less than $\beta$.

Suppose the multiplication rule in $H$ is given by $e^{(\alpha)} e^{(\beta)}=\sum_{\mu} \gamma_{\mu}^{\alpha, \beta} e^{(\mu)}$. It is also useful to set $\gamma_{\mu}^{\alpha, \beta}=0$ if either of $\alpha, \beta, \mu$ contains a negative component.

The standard coproduct on $H$ is easy to compute in this notation: $\Delta\left(e^{(\alpha)}\right)=\sum_{\nu} e^{(\alpha-\nu)} \otimes e^{(\nu)}$.

Theorem 3. Let $J$ be a simple finite Jordan pseudoalgebra over $H=U(\mathfrak{h})$, where $\mathfrak{h}$ is a finitedimensional Lie algebra over the field $\mathbb{C}$. Then $J$ is isomorphic to the current algebra $\mathrm{Cur} \mathfrak{j}$ over a finite-dimensional simple Jordan algebra $\mathfrak{j}$.

Lemma 6. Let $C=\operatorname{Cur} \mathfrak{g}=H \otimes \mathfrak{g}$. Consider an arbitrary pair of elements $a, b \in C, a=$ $\sum_{\alpha} e^{(\alpha)} \otimes a_{\alpha}, b=\sum_{\beta} e^{(\beta)} \otimes b_{\beta}, a_{\alpha}, b_{\beta} \in \mathfrak{g}$. If $[a * b]=0$, then $\left[a_{\alpha} b_{\beta}\right]=0$ in $\mathfrak{g}$ for all $\alpha, \beta$. 
Proof. Straightforward computations show that

$$
[a * b]=\sum_{\alpha, \beta, \nu, \mu}(-1)^{|\beta-\nu|} \gamma_{\mu}^{\alpha, \beta-\nu}\left(e^{(\mu)} \otimes 1\right) \otimes_{H}\left(e^{(\nu)} \otimes\left[a_{\alpha} b_{\beta}\right]\right) .
$$

If $[a * b]=0$ then (4.1) implies that for every $\nu, \mu$ we have

$$
\sum_{\alpha, \beta}(-1)^{|\beta-\nu|} \gamma_{\mu}^{\alpha, \beta-\nu}\left[a_{\alpha} b_{\beta}\right]=0
$$

Put $\nu=\beta^{\max }$ in (4.2) (i.e., $b_{\nu} \neq 0$, but $b_{\beta}=0$ for all $\beta>\nu$ ). We obtain $\sum_{\alpha} \gamma_{\nu}^{\alpha, 0}\left[a_{\alpha} b_{\nu}\right]=0$ for each $\mu$. However,

$$
\gamma_{\mu}^{\alpha, 0}= \begin{cases}0, & \mu \neq \alpha, \\ 1, & \mu=\alpha,\end{cases}
$$

hence, $\left[a_{\alpha} b_{\beta_{\max }}\right]=0$ for each $\alpha$.

To finish the proof, use the induction on $\beta$. Suppose that $\left[a_{\mu} b_{\beta}\right]=0$ for all $\mu$ and $\beta>\beta_{0}$. Let us show that $\left[a_{\mu} b_{\beta_{0}}\right]=0$. Put $\nu=\beta_{0}$. Relation (4.2) implies $0=\sum_{\alpha} \gamma_{\mu}^{\alpha, 0}\left[a_{\alpha} b_{\beta_{0}}\right]+$ $\sum_{\beta>\beta_{0}}(-1)^{|\nu-\beta|} \gamma_{\mu}^{\alpha, \beta-\nu}\left[a_{\alpha} b_{\beta}\right]$. The second summand is equal to zero by the inductive assumption. So we have $\left[a_{\alpha} b_{\beta_{0}}\right]=0$ for each $\alpha$.

Now, let $\mathcal{L}=J^{+} \oplus \mathrm{S}_{0}(J) \oplus J^{-}=$Cur $\mathfrak{g} . \quad$ By $\mathfrak{j}_{0}^{ \pm}$we denote the spaces spanned by all coefficients $a_{\alpha} \in \mathfrak{g}$ ingoing in the sums $\sum_{\alpha} e^{(\alpha)} \otimes a_{\alpha} \in J^{ \pm}$. Lemma 6 implies the spaces $\mathfrak{j}_{0}^{ \pm}$are Abelian subalgebras of $\mathfrak{g}$ such that $\left[H \otimes \mathfrak{j}_{0}^{ \pm} * J^{ \pm}\right]=0$. Moreover, $H \otimes \mathfrak{j}_{0}^{ \pm} \supseteq J^{ \pm}$.

Lemma 7. Let $\mathcal{L}=\mathrm{T}(J)=\mathrm{Cur} \mathfrak{g}$, where $\mathfrak{g}$ is a finite-dimensional Lie algebra. Suppose that there are no non-zero ideals $I \triangleleft \mathcal{L}$ such that $\pi^{ \pm}(I)=0$. Then $J^{ \pm}=H \otimes \mathfrak{j}_{0}^{ \pm}$, respectively.

Proof. It is enough to consider the "+" case. Consider an arbitrary element $a \in H \otimes \mathfrak{j}_{0}^{+}$, $a=\pi_{+}(a)+\pi_{0}(a)+\pi_{-}(a)$. Denote $J_{0}^{-}=\pi_{-}\left(H \otimes \mathfrak{j}_{0}^{+}\right), J_{0}^{0}=\pi_{0}\left(H \otimes \mathfrak{j}_{0}^{+}\right)$.

For every $b \in J^{+}$we have $0=[a * b]=\left[\pi_{+}(a) * b\right]+\left[\pi_{0}(a) * b\right]+\left[\pi_{-}(a) * b\right]$. Since $\left[\pi_{0}(a) * b\right] \in$ $H^{\otimes 2} \otimes_{H} \mathrm{~S}_{0}(J),\left[\pi_{-}(a) * b\right] \in H^{\otimes 2} \otimes_{H} J^{+},\left[\pi_{+}(a) * b\right]=0$, then

$$
\left[J_{0}^{-} * J^{+}\right]=\left[J_{0}^{0} * J^{+}\right]=0 .
$$

Given $H$-submodules $A, B \subseteq \mathcal{L}$, denote by $[A \cdot B] \subseteq \mathcal{L}$ the $H$-module spanned (over $\mathbb{C}$ ) by all Fourier coefficients of all elements from $[A * B]$. By $\left[A^{\omega} \cdot B\right]$ we denote the sum of $H$-modules $\sum_{n \geq 0}\left[A^{n} \cdot B\right]$, where $\left[A^{0} \cdot B\right]=B,\left[A^{n+1} \cdot B\right]=\left[A \cdot\left[A^{n} \cdot B\right]\right]$.

For example, $\left[\mathrm{S}_{0}(J)^{\omega} \cdot J_{0}^{-}\right] \subseteq J^{-}$. Moreover, the Jacobi identity and (4.3) imply $\left[J^{+} *\left[\mathrm{~S}_{0}(J)^{\omega}\right.\right.$. $\left.\left.J_{0}^{-}\right]\right]=0$. It is also easy to note that $\left[\mathrm{S}_{0}(J) *\left[\mathrm{~S}_{0}(J)^{\omega} \cdot J_{0}^{-}\right]\right] \subseteq H^{\otimes 2} \otimes_{H}\left[\mathrm{~S}_{0}(J)^{\omega} \cdot J_{0}^{-}\right]$. Since $\left[J^{-} *\left[\mathrm{~S}_{0}(J)^{\omega} \cdot J_{0}^{-}\right]\right]=0$, then $I=\left[\mathrm{S}_{0}(J)^{\omega} \cdot J_{0}^{-}\right]$is a proper ideal of $\mathcal{L}, I \supseteq J_{0}^{-}$and $\pi^{+}(I)=0$. Hence, $I=0$, and $J_{0}^{-}=0$.

Further, let us consider

$$
I=\left[\mathrm{S}_{0}(J)^{\omega} \cdot J_{0}^{0}\right]+\left[\mathrm{S}_{0}(J)^{\omega} \cdot\left[J^{-} \cdot J_{0}^{0}\right]\right] \subseteq \mathrm{S}_{0}(J) \oplus J^{-} .
$$

It follows from (4.3) that $\left[J^{+} *\left[\mathrm{~S}_{0}(J)^{\omega} \cdot J_{0}^{0}\right]\right]=0$. Moreover, $\left[J^{+} *\left[\mathrm{~S}_{0}(J)^{\omega} \cdot\left[J^{-} \cdot J_{0}^{0}\right]\right]\right] \subseteq$ $\left.H^{\otimes 2} \otimes_{H}\left[\mathrm{~S}_{0}(J)^{\omega} \cdot J_{0}^{0}\right]\right]$. Therefore, $\left[J^{+} \cdot I\right] \subseteq I$. Since $\left[\mathrm{S}_{0}(J) \cdot I\right] \subseteq I$ by construction, and $\left[J^{-} \cdot I\right] \subseteq I$ by the Jacobi identity, the ideal (4.4) is proper in $\mathcal{L}$, so $J_{0}^{0}=0$.

We have proved that $\pi_{-}\left(H \otimes \mathfrak{j}_{0}^{+}\right)=\pi_{0}\left(H \otimes \mathfrak{j}_{0}^{+}\right)=0$. Thus, $J^{+}=H \otimes \mathfrak{j}_{0}^{+}$. 
Hence, under the conditions of Lemma 7 one has $J=H \otimes \mathfrak{j}, \mathfrak{j} \simeq \mathfrak{j}_{0}^{ \pm}$. Now it is necessary to show that the Jordan pseudoproduct on $J$ may be restricted to an ordinary Jordan product on $\mathfrak{j}$.

Proposition 4. Let $J$ be a finite Jordan H-pseudoalgebra such that $\operatorname{Ann}_{l}(J):=\{a \in J \mid a * J=$ $0\}=0$. Assume that $J=H \otimes \mathfrak{j}$, where $\mathfrak{j}$ is a linear space. If $\mathcal{L}=\mathrm{T}(J)=$ Cur $\mathfrak{g}$ then $\mathfrak{j}$ has a structure of ordinary Jordan algebra such that $\mathfrak{g} \simeq \mathrm{T}(\mathfrak{j})$.

Proof. Let $a, b \in J$ be some elements of the form $a=1 \otimes \alpha, b=1 \otimes \beta, \alpha, \beta \in \mathfrak{j}$. Then $2 L_{a * b}=\left[a^{-} * b^{+}\right]+\left(\sigma_{12} \otimes_{H}\right.$ id $)\left[b^{-} * a^{+}\right]=(1 \otimes 1) \otimes_{H}\left(1 \otimes\left[\alpha^{-} \beta^{+}\right]+1 \otimes\left[\beta^{-} \alpha^{+}\right]\right)$(here $\alpha^{ \pm}$denote the images of $\alpha \in \mathfrak{j}$ in $\left.\mathfrak{j}_{0}^{ \pm}\right)$.

Thus, $L_{a * b}=(1 \otimes 1) \otimes_{H}(1 \otimes s(\alpha, \beta)) \in \mathcal{L}$, where $s(\alpha, \beta) \in\left[\mathfrak{j}^{-} \mathfrak{j}^{+}\right] \subseteq \mathfrak{g}$. Therefore, $L_{\left(a \circ_{t^{\nu}} b\right)}=0$ for $\nu=\left(\nu_{1}, \ldots, \nu_{n}\right)>(0, \ldots, 0)$. Here we have used the notation $t^{\nu}=t_{1}^{\nu_{1}} \ldots t_{n}^{\nu_{n}}$ for basic functionals in $X=H^{*}$.

Since $L_{x}=0$ implies $x=0$, we have

$$
a * b=(1 \otimes 1) \otimes_{H} c, \quad c \in J .
$$

Suppose that $c=\sum_{\mu} e^{(\mu)} \otimes \gamma_{\mu}, \gamma_{\mu} \in \mathfrak{j}$. Assume that the maximal $\mu=\mu_{\max }$ such that $\gamma_{\mu} \neq 0$ is a multi-index greater than $(0, \ldots, 0)$. Then

$$
\left[(1 \otimes s(\alpha, \beta)) \circ_{t^{\mu} \max }\left(1 \otimes \delta^{-}\right)\right]=0
$$

for all $\delta \in \mathfrak{j}$. On the other hand, $\left[(1 \otimes s(\alpha, \beta)) \circ_{t^{\mu} \max }\left(1 \otimes \delta^{-}\right)\right]=\left(c \circ_{t^{\mu}}{ }_{\max }(1 \otimes \delta)\right)^{-}$. It is easy to see that the relations (4.5), (4.6) and the axioms of a pseudoalgebra imply $\left(c \circ_{t^{\mu}}{ }_{\max }(1 \otimes \delta)\right)=$ $\left(\left(1 \otimes \gamma_{\mu_{\max }}\right)_{\circ_{\varepsilon}}(1 \otimes \delta)\right)=0$, i.e., $L_{1 \otimes \gamma_{\mu_{\max }}}=0$. Thus, $\gamma_{\mu_{\max }}=0$, which is a contradiction.

We have proved that $(1 \otimes \alpha) *(1 \otimes \beta)=(1 \otimes 1) \otimes_{H}(1 \otimes \gamma(\alpha, \beta)), \gamma(\alpha, \beta) \in \mathfrak{j}$. This relation leads to an ordinary product on $\mathfrak{j}$ defined by the rule $\alpha \cdot \beta=\gamma(\alpha, \beta)$. Then the pseudoalgebra $J$ is a current pseudoalgebra over $\mathfrak{j}$, and $(\mathfrak{j}, \cdot)$ is necessarily a simple finite-dimensional Jordan algebra. To complete the proof, it is enough to note that $T(\mathrm{Cur} \mathfrak{j}) \simeq \mathrm{Cur} T(\mathfrak{j})$. For finite-dimensional Lie algebras $\mathfrak{g}_{1}, \mathfrak{g}_{2}$ the condition Cur $\mathfrak{g}_{1} \simeq$ Cur $\mathfrak{g}_{2}$ implies $\mathfrak{g}_{1} \simeq \mathfrak{g}_{2}$.

Proof of Theorem 3. Let $J$ be a simple finite Jordan pseudoalgebra. Proposition 3 implies that $\mathcal{L}=\mathrm{T}(J) \simeq$ Cur $\mathfrak{g}$, where $\mathfrak{g}$ is a simple finite-dimensional Lie algebra. By Lemma 7 , $J=H \otimes \mathfrak{j}$. Since $\mathcal{L}$ satisfies the conditions of Proposition 4 , we have $J \simeq \operatorname{Cur} \mathfrak{j}, \mathrm{T}(\mathfrak{j})=\mathfrak{g}$, where $\mathfrak{j}$ is a simple finite-dimensional Jordan algebra.

Corollary 1 ([19]). A simple finite Jordan conformal algebra is isomorphic to the current conformal algebra over a simple finite-dimensional Jordan algebra.

\subsection{The case $\boldsymbol{H}=\boldsymbol{U}(\mathfrak{h}) \# \mathbb{C}[\Gamma]$}

If $J$ is a pseudoalgebra over $H=U(\mathfrak{h}) \# \mathbb{C}[\Gamma]$ then it is in particular a pseudoalgebra over $U(\mathfrak{h})$. The structure of $H$-pseudoalgebra on $J$ is completely encoded by $U(\mathfrak{h})$-pseudoalgebra structure and by the action of $\Gamma$ on $U(\mathfrak{h})$, see [1, Section 5] for details.

Theorem 4. Let $J$ be a simple Jordan pseudoalgebra over $H=U(\mathfrak{h}) \# \mathbb{C}[\Gamma], \operatorname{dim} \mathfrak{h}<\infty$, which is a finitely generated $U(\mathfrak{h})$-module. Then

$$
J \simeq \bigoplus_{i=1}^{m} \operatorname{Cur}^{U(\mathfrak{h})} \mathfrak{j}_{i},
$$

where $\mathfrak{j}_{i}$ are isomorphic finite-dimensional simple Jordan algebras, and $\Gamma$ acts transitively on the family $\left\{\operatorname{Cur}^{U(\mathfrak{h})} \mathfrak{j}_{i}: i=1, \ldots, m\right\}$. 
Proof. By Proposition $2 \mathcal{L}=\mathrm{T}(J)$ is a simple $H$-pseudoalgebra, and it is clear that $\mathcal{L}$ is a finitely generated $U(\mathfrak{h})$-module. Theorem 2 and Proposition 3 imply that $\mathcal{L}=\bigoplus_{i=1}^{m} \operatorname{Cur}^{U(\mathfrak{h})} \mathfrak{g}_{i}$ where $\operatorname{Cur}^{U(\mathfrak{h})} \mathfrak{g}_{i}=\operatorname{Cur}_{i}$ are isomorphic simple current Lie $U(\mathfrak{h})$-pseudoalgebras, and $\Gamma$ acts on them transitively.

Hence, $\mathcal{L}=\operatorname{Cur}^{U(\mathfrak{h})} \tilde{\mathfrak{g}}$, where $\tilde{\mathfrak{g}}=\bigoplus_{i=1}^{m} \mathfrak{g}_{i}$. The $H$-pseudoalgebra $\mathcal{L}$ could be considered as an $U(\mathfrak{h})$-pseudoalgebra endowed with an action of $\Gamma$ on it which is compatible with that of $U(\mathfrak{h})$ : $g(h a)=h^{g}(g a), h \in U(\mathfrak{h}), a \in \mathcal{L}, g \in \Gamma$.

Consider $\mathcal{L}$ as the current $U(\mathfrak{h})$-pseudoalgebra over $\tilde{\mathfrak{g}}$. The condition of Lemma 7 holds for this $\mathcal{L}$. Indeed, if $I$ is an ideal of the $U(\mathfrak{h})$-pseudoalgebra $\mathcal{L}$ and $\pi^{ \pm}(I)=0$, then $\Gamma I$ is a proper ideal of $\mathcal{L}$ (as of an $H$-pseudoalgebra) such that its projections onto $J^{ \pm}$are zero. Moreover, if $J$ as an $H$-pseudoalgebra has no non-trivial (left) annihilator $\operatorname{Ann}_{l}(J)$ then so is $J$ as an $U(\mathfrak{h})$-pseudoalgebra (see [1, Corollary 5.1]).

Therefore, the same arguments as in the proof of Proposition 4 show that $J=\operatorname{Cur}^{U(\mathfrak{h})} \tilde{\mathfrak{j}}$, where $\tilde{\mathfrak{j}}$ is a finite-dimensional Jordan algebra.

The explicit expression [1, equation (5.7)] for pseudoproduct over $H$ shows that for every $x \in X=U(\mathfrak{h})^{*}, g \in \Gamma, a, b \in J$ we have

$$
\left(a \circ_{x \otimes g^{*}} b\right)=\left(a \circ_{(x \otimes 1) g} b\right)=\left(g a \circ_{x} b\right),
$$

where $\left\langle g^{*}, \gamma\right\rangle=\delta_{g, \gamma}, \gamma \in \Gamma$. Hence, the following relation between Fourier coefficients of $U_{a, b}$ holds: $U_{\left(a \circ_{\left.x \otimes g^{*} b\right)}\right.}=U_{\left(g a \circ_{x} b\right)}$. Here in the left- and right-hand sides we state Fourier coefficients over $H^{*}$ and $X$, respectively. Therefore, the relations between the $H$-module $\mathrm{S}_{0}(H J)$ and the $U(\mathfrak{h})$-module $U(\mathfrak{h}) \mathrm{S}_{0}(J)$ are the same as between $H$-module ${ }_{H} J$ and $U(\mathfrak{h})$-module $U(\mathfrak{h}) J$.

Now it is clear that $\tilde{\mathfrak{g}}=\mathrm{T}(\tilde{\mathfrak{j}})$. Hence, $\tilde{\mathfrak{j}}=\bigoplus_{i=1}^{m} \mathfrak{j}_{i}, \mathfrak{g}_{i}=\mathrm{T}\left(\mathfrak{j}_{i}\right)$, where $\mathfrak{j}_{i}$ are simple Jordan algebras. So, $J=\bigoplus_{i=1}^{m} \operatorname{Cur}^{U(\mathfrak{h})} \mathfrak{j}_{i}$, and $\Gamma$ necessarily acts on these current algebras transitively.

\section{Acknowledgements}

I am very grateful to L.A. Bokut, I.V. L'vov, E.I. Zel'manov, and V.N. Zhelyabin for their interest in the present work and helpful discussions. It is my pleasure to appreciate the efforts of the referees, whose suggestions and comments helped me to make the paper readable.

The work was partially supported by SSc-344.2008.1. I gratefully acknowledge the support of the Pierre Deligne fund based on his 2004 Balzan prize in mathematics, and Novosibirsk City Administration grant of 2008.

\section{References}

[1] Bakalov B., D'Andrea A., Kac V.G., Theory of finite pseudoalgebras, Adv. Math. 162 (2001), 1-140, math.QA/0007121.

[2] Beilinson A.A., Drinfeld V.G., Chiral algebras, American Mathematical Society Colloquium Publications, Vol. 51, American Mathematical Society, Providence, RI, 2004.

[3] Belavin A.A., Polyakov A.M., Zamolodchikov A.B., Infinite conformal symmetry in two-dimensional quantum field theory, Nuclear Phys. B 241 (1984), 333-380.

[4] Borcherds R.E., Vertex algebras, Kac-Moody algebras, and the Monster, Proc. Nat. Acad. Sci. U.S.A. 83 (1986), 3068-3071.

[5] D'Andrea A., Kac V.G., Structure theory of finite conformal algebras, Selecta Math. (N.S.) 4 (1998), 377418. 
[6] Fattori D., Kac V.G., Classification of finite simple Lie conformal superalgebras, J. Algebra 258 (2002), 23-59, math-ph/0106002.

[7] Kac V.G., Vertex algebras for beginners, 2nd ed., University Lecture Series, Vol. 10, American Mathematical Society, Providence, RI, 1998.

[8] Kac V.G., Formal distribution algebras and conformal algebras, in Proceedinds of XII-th International Congress in Mathematical Physics (ICMP'97) (Brisbane), Int. Press, Cambridge, MA, 1999, 80-97, q-alg/9709027.

[9] Kac V.G., Retakh A., Simple Jordan conformal superalgebras, J. Algebra Appl. 7 (2008), 517-533, arXiv:0801.0755.

[10] Kantor I.L., Classification of irreducible transitively differential groups, Soviet Math. Dokl. 5 (1965), 14041407.

[11] Koecher M., Embedding of Jordan algebras into Lie algebras. I, Amer. J. Math. 89 (1967), 787-816.

[12] Koecher M., Embedding of Jordan algebras into Lie algebras. II, Amer. J. Math. 90 (1968), 476-510.

[13] Kolesnikov P.S., Identities of conformal algebras and pseudoalgebras, Comm. Algebra 34 (2006), no. 6, 1965-1979, math.RA/0412397.

[14] Lambek J., Deductive systems and categories II. Standard constructions and closed categories, in Category Theory, Homology Theory and their Applications, I (Battelle Institute Conference, Seattle, Wash., 1968, Vol. One), Springer, Berlin, 1969, 76-122.

[15] Roitman M., On free conformal and vertex algebras, J. Algebra 217 (1999), 496-527, math.QA/9809050.

[16] Sweedler M.E., Cocommutative Hopf algebras with antipode, Bull. Amer. Math. Soc. 73 (1967), $126-128$.

[17] Sweedler M.E., Hopf algebras, Mathematics Lecture Note Series, W.A. Benjamin, Inc., New York, 1969.

[18] Tits J., Une classe d'algebres de Lie en relation avec algebres de Jordan, Indag. Math. 24 (1962), 530-535.

[19] Zelmanov E.I., On the structure of conformal algebras, in Proceedings of Intern. Conf. on Combinatorial and Computational Algebra, (May 24-29, 1999, Hong Kong) Contemp. Math 264 (2000), 139-153.

[20] Zhevlakov K.A., Slin'ko A.M., Shestakov I.P., Shirshov A.I., Rings that are nearly associative, Pure and Applied Mathematics, Vol. 104, Academic Press, Inc., New York - London, 1982. 